\title{
Activity and Fitness in Spinal Cord Injury: Review and Update
}

\author{
Sue Ann Sisto • Nick Evans
}

Published online: 11 July 2014

(C) Springer Science + Business Media New York 2014

\begin{abstract}
The primary aims of this report are to provide a review of current literature pertaining to fitness and healthrelated outcomes following spinal cord injury (SCI), to identify the common and relevant fitness assessment techniques, to describe key evidence-based intervention strategies for improving health-related components of fitness among persons with SCI and to identify emerging and future trends in health-related fitness programming for rehabilitation and research. SCI results in a partial or total loss of neural signal transmission at and below the level of injury, characterized by motor and sensory loss. About 250,000-400,000 individuals have a SCI in the USA with approximately 12,000 new injuries occurring annually. The loss of somatic and autonomic control results in a reduction of physical activity and blunted cardiovascular response to exercise. The consequences of this reduction in physical activity are significant physical deconditioning, altered body composition, and development of detrimental metabolic profiles leading to poorer health outcomes in this population. Over the past decade, much attention has been devoted to understanding the relationship between exercise participation, physical activity, and physical fitness and their impact on health-related outcomes following SCI. Physical fitness
\end{abstract}

\section{S. A. Sisto $(\square)$}

School of Health Technology and Management, Research and Development Park, Rehabilitation Research and Movement Performance (RRAMP) Laboratory, Development Drive, Suite 120, Stony Brook University, Stony Brook, NY 11794-6018, USA

e-mail: sue.sisto@ stonybrook.edu

\section{N. Evans}

Virginia C Crawford Research Institute, Shepherd Center, Atlanta, GA, USA

e-mail: nicholas_evans@shepherd.org interventions are of great importance to the SCI consumer and clinicians whose goals are to halt the deconditioning process, increase functional capacity, and decrease secondary health complications associated with chronic SCI. Exercise risk evaluation and individualized fitness assessments for body composition, muscular strength/endurance, and cardiorespiratory fitness should precede implementation of exercise interventions. Current evidence indicates that both electrically stimulated and volitional exercise strategies have the potential to improve components of fitness, reduce the risk of secondary health complications, and positively impact overall health for those with chronic physical disability. However, given the complexity of SCI with respect to level and severity of injury, a clear consensus on the optimal frequency, duration, and intensity of exercise needed to ensure lasting effects on health-related outcomes has yet to be determined. In addition, as is the case in non-injured populations, consideration should be given to both diet and exercise, not exercise alone, when considering the optimal strategy for improving body composition and cardiovascular health for persons with SCI.

Keywords Spinal cord injuries - Exercise $\cdot$ Physical fitness $\cdot$ Health $\cdot$ Human physical conditioning

\section{Introduction}

Spinal cord injury (SCI) results in a partial or total loss of neural signal transmission at and below the level of injury, characterized by motor and sensory loss. About 250,000-400,000 individuals have a SCI in the USA with approximately 12,000 new injuries occurring annually [1]. The loss of somatic and autonomic control results in a reduction of physical activity and blunted cardiovascular 
response to exercise. Consequences of this reduction in physical activity include detrimental changes in body composition [2] and metabolic profiles [3, 4] leading to significantly poorer health outcomes in this population. The World Health Organization's International Classification of Functioning, Disability and Health (ICF) provides a valuable framework for understanding the complex interaction between the various factors that can affect impairments (fitness), function (activity), and participation (barriers/ facilitators to community-based fitness) after a SCI [5, 6]. Secondary health conditions are indirectly related to the primary deficit (e.g., SCI and spasticity) and result in conditions such as obesity, cardiovascular disease, reduced pulmonary capacity, and deconditioning. We will address these conditions, and their impact on fitness and activity.

Obesity is a major public health concern and is more likely to exist among populations where physical inactivity, sedentary behavior, and poor dietary habits are widespread. Obesity is exacerbated in SCI due to reduced capacity to incorporate all muscles for exercise benefits resulting in the dramatic loss of muscle mass and an increase in whole body and regional adiposity. This accumulation of fat mass can interfere with insulin and lipids metabolism, resulting in detrimental levels of circulating lipids (high level of triglycerides and low levels of high density lipoprotein cholesterol (HDLc), known to be risk factors for cardiovascular disease, type 2 diabetes, and metabolic syndrome. The prevalence of metabolic syndrome, defined as having central obesity (increased visceral fat mass), insulin resistance, hypertension, and dyslipidemia, is reported to be between 32 and $34 \%$ in individuals with SCI [7, 8]. The prevalence is higher ( $45 \%$ ) in individuals with tetraplegia [9] than those with paraplegia. True fat mass in individuals with SCI-related paralysis can exceed $30 \%$ despite calculated body mass index (BMI) being less than $30 \mathrm{~kg} \mathrm{~m}^{-2}$, as the method of calculating BMI in able-bodied individuals underestimates true fat mass in individuals with SCIrelated paralysis [10]. Amount of visceral fat mass inversely correlates with HDLc levels and directly correlates with triglycerides levels (TG) [11].

Individuals with SCI demonstrate reduced pulmonary capacity; the pulmonary capacity inversely correlates with the neurological level of injury (NLI). Although there have been improvements in medical care following SCI, pneumonia and other respiratory illnesses remain number one cause of morbidity and mortality following paralysis related to spinal cord injury $[12,13]$ In one study, $12 \%$ of 99 veterans with SCI reported respiratory problems as their principal secondary medical problem after SCI [14]. Pulmonary complications in patients with chronic cervical and upper thoracic SCI are related to the persistent respiratory muscle weakness and paralysis [15]. Inability to facilitate an effective exhalation and cough due to expiratory muscle weakness leads to development of very serious pulmonary complications associated with an accumulation of secretions, airway obstruction, and pneumonia [16]. Spastic contractions of the respiratory muscles reduce the elastic properties of the respiratory system causing poor ventilation and inducing a form of restrictive lung disease [17]. Exercise programs can increase fitness and improve the ventilatory function in individuals with acute [18] and chronic SCI [19]. It has been shown that exercise in individuals with chronic SCI elicits a metabolic response characterized by increased oxygen consumption and minute ventilation [20].

\section{Physical Deconditioning Following SCI}

Physical deconditioning is a state of diminished strength, stamina, and capacity to perform physical activity. A decline in strength, endurance, and functional capacity are major health concerns for the individual living with SCI. As stated before, sedentary behavior and inactivity are major risk factors for the development of cardiovascular disease and metabolic disorders and have been linked to decreased muscular strength, reduced aerobic capacity, and increased disability [21, 22].

Depending on the severity and level of injury, paralysis of the limb and trunk muscles results in immediate and permanent changes in muscle morphology and physiology. In subacute and chronic SCI, a cascade of changes occur within affected skeletal muscle including atrophy of both type I and type II fibers, transformation from type I and IIA to type IIX, loss of contractile proteins, and decreased oxidative enzymatic activity [23-26]. As time post-injury increases, these altered muscle characteristics result in decreased muscle strength and resistance to fatigue adding to the systemic physical deconditioning [23].

A decline in cardiovascular function has also been observed in SCI and can significantly contribute to deconditioning and reduced functional capacity following the injury. It is well documented that cardiovascular control is altered by disruption of descending input to the autonomic nervous system (ANS), especially with cervical injuries. Disordered ANS input can have a significant effect on blood pressure, heart rate, and temperature regulation. This dysfunction ultimately leads to an inability to challenge the cardiovascular system to the extent required to achieve health and fitness benefits [27-29••]. Couple these cardiovascular impairments with reduced pulmonary function caused by weakness and stiffness of the respiratory muscles, and the ability to effectively engage in physical activity is further compromised [30, 31].

Using the ICF framework, we can understand the complex interaction between the various factors that might 
affect an individuals' likelihood or ability to engage in exercise or leisure time related physical activity [6]. The role of psychosocial and environmental factors must be considered in the context of deconditioning following SCI as the likelihood that an individual will feel compelled to or have the ability to participate in activities are highly dependent on these factors. Current research has demonstrated that exercise participation among those with mobility impairments is low and sedentary time is high in this population [32-34]. Structured leisure time physical activity and exercise participation has been attributed to the existence of unique environmental, social, and psychological barriers experienced by the individual [33, 35-37]. A combination of barriers might limit the extent to which someone becomes or remains physically active. It is important to understand this complex interaction between the individual, their environment, and their perceived barriers in order to develop effective strategies to overcome these obstacles and to promote healthy lifestyle choices.

Changes in cardiopulmonary and muscle physiology as well as the existence of barriers to exercise play a major role in the deconditioning process following SCI. These factors contribute to total body physical decline, impaired physical capacity, decreased independence performing activities of daily living, and increased risk for the development of cardiovascular disease and metabolic dysfunction. Thus, physical deconditioning is of major concern for those living with SCI and should be addressed by targeted evidence-based interventions with an emphasis on those interventions that have measurable health-related fitness outcomes.

\section{Fitness and Spinal Cord Injury}

Over the past decade, much attention has been devoted to understanding the relationship between exercise participation, physical activity, and physical fitness and their impact on health-related outcomes following SCI [32, 33, 38-42]. Physical fitness consists of a specific set of attributes that an individual may have or may achieve that enhances the ability to carry out daily tasks without undue fatigue and with ample energy [43, 44]. Physical fitness interventions are of great importance to the consumer and clinicians whose goals are to halt the deconditioning process, increase functional capacity, and decrease secondary health complications associated with chronic SCI. Evidence indicates that strategies to promote exercise and improve the components of fitness positively affect the overall health of individuals with SCI and can reduce the risk of secondary health complications associated with chronic disability [32, 40-42, 45].

There are five health-related components of physical fitness, four of which will be the focus of this review (body composition, cardiovascular endurance, muscular strength, and muscular endurance). Although flexibility and the ability to move a joint through its full range of motion is important to overall mobility, we will limit our discussion to those health-related fitness attributes that, in their absence, have been more closely associated with increased morbidity and mortality in SCI.

\section{Body Composition}

Body composition can be used to provide health professionals with critical information concerning individual physical fitness, metabolic health, and relative risk for developing type 2 diabetes mellitus, hypertension, hyperlipidemia, cardiovascular disease, and certain types of cancers. Measurement of body composition provides a means of quantifying total body adiposity and can help objectively identify the presence of obesity wherein specific threshold values are either met or exceeded (e.g., body fat $>30 \%$ [females], body fat $>25 \%$ [males]). The physical, metabolic, and lifestyle changes that occur following a SCI result in lower values of total daily energy expenditure, reduced resting metabolic rate, reduced thermic effect of activity, and reduced thermic effect of food even after adjustment of fatfree mass $[2,46]$. The consequence of these combined factors is a higher prevalence of obesity in SCI, and thus, a greater risk for the development of comorbidities compared to noninjured people [2, 47, 48].

\section{Body Composition Assessment Methods}

Determining body fat percentage has clinical relevance and value, and various field and laboratory techniques have been developed to estimate body composition. Each of these techniques varies in terms of cost, complexity, reliability, and validity. Body composition is estimated by partitioning total body mass into fat mass and fat-free mass (e.g., muscle, bone, connective tissue, internal organs, water, protein, and mineral composition). Basic field assessments of body composition can be performed relatively quickly, require minimal equipment, and include measures such as body mass index (BMI), circumference measures, bioelectrical impedance (BIA), near-infrared interactance (NIR), and skinfold measures (SKF). More advanced laboratory assessments, often found in clinical and research settings, can been used to estimate body composition and may provide greater accuracy when compared to field tests but require specialized equipment, may be costly to perform, and are not easily accessed by exercise professionals and consumers. Laboratory assessments for estimating body composition include hydrostatic weighing, air displacement plethysmography, computed 
tomography (CT) scans, magnetic resonance imaging (MRI), ultrasound, and dual-energy X-ray absorptiometry (DXA). DXA is not the least expensive assessment option, but it appears to meet reliability and validity standards for clinical populations, is easy to administer, and provides a comfortable experience for users compared with other laboratory techniques.

Some body composition assessment methods are based on specific assumptions regarding fat-free composition that may not be valid in SCI [49]. For more than a decade, a number of studies have investigated the validity of field measures such as SKF, BIA, and BMI against more robust clinical measures such as DXA and have found, consistently, that field assessments of body composition significantly underestimate body fat percentage in the SCI population and that predictive equations developed for non-injured populations are a poor fit for those with SCI [50-54]. Thus, clinicians should consider the accuracy of these measures when used in SCI given the well documented changes in their fat and fat-free mass [24, 55-58].

\section{Body Composition Intervention Strategies}

Intervention strategies for optimizing body composition are well established in the literature for non-clinical populations [59]. The most effective strategies involve a combination of dietary interventions, aerobic exercise, resistance training, and behavioral counseling. Although there are many complex physiologic, metabolic, and biochemical mechanisms that mediate changes in body composition, at the most basic level, the aims of dietary and exercise interventions are to increase daily energy expenditure, decrease daily caloric intake, and increase lean muscle mass as metabolically active tissue. Following a SCI, dietary habits are often altered and the inability to perform compound exercises using large muscle groups makes it more challenging to achieve an ideal caloric balance to avoid accumulation of excess fat. The benefits of exercise participation for body composition management can be realized by persons with SCI $[38,60]$. Electrically stimulated resistance training has been reported to alter body composition by increasing lean muscle mass after SCI [61-63]. However, despite positive changes observed in lean muscle mass, there is conflicting evidence as to the extent to whether electrically stimulated resistance training on its own can positively impact adiposity in this population $[64,65]$.

In addition to electrical stimulation, participation in volitional physical activity and exercise has also been shown to improve body composition with a potentially greater effect on regional fat mass when compared to electrical stimulation alone. Individuals with tetraplegia who participated in at least 150 min per week of physical activity had significantly lower total and regional fat mass compare to individuals with tetraplegia who were not physically active [38]. In individuals with paraplegia, 8 weeks of upper extremity resistance training resulted in an increase in upper extremity fat-free mass and a decrease in fat mass [66]. Despite signs pointing toward the benefits of volitional and electrically stimulated exercise for improving body composition in SCI, a clear consensus on the type, frequency, duration and intensity of exercise needed to achieve clinically meaningful improvements has yet to be determined [67]. In addition, there is a lack of understanding of the role of combined intervention strategies such as exercise, diet modification, and behavioral counseling on body composition and SCI [68]. Further research is needed to understand the complex interactions between multiple intervention strategies and body composition following SCI.

\section{Muscular Strength/Endurance}

Muscular strength and muscular endurance are important health-related components of fitness and play a vital role in improving or maintaining bone mineral density, fat-free mass, resting metabolic rate, muscle force production and power, musculotendinous integrity, and glucose metabolism [43]. Muscular strength and endurance constitute two components of muscular fitness. Muscle strength refers to the ability of a muscle or muscle group to generate maximal force, and muscle endurance refers to the ability of a muscle or muscle group to produce force over multiple repetitions [43]. Both components can be significantly impaired as a result of SCI. Mitigating the deleterious effects of muscle deconditioning is a major priority for clinicians and exercise professionals working with persons with SCI and, in fact, is the primary goal for most SCI rehabilitation programs.

\section{Muscle Strength and Endurance Assessment Methods}

Muscular strength and endurance testing can provide valuable information regarding baseline fitness levels. However, because of the variability of SCI level and severity, norm-reference outcomes for muscular strength and endurance have been difficult to establish. Therefore, it is recommended that testing of muscular strength and endurance should be used to compare changes within an individual versus between individuals. A number of tests of muscular strength and endurance for persons with SCI have been utilized and include 1-repetition maximum (1RM), 10-repetition maximum (10RM), isokinetic, isometric, and manually facilitated testing. Lower extremity dynamometers are often found in sports medicine, physical therapy clinics and research settings, can be expensive to acquire, 
and may not be applicable to individuals who lack sufficient lower extremity power to move the crank arm. However, for individuals with sufficient spared volitional lower extremity power (e.g., motor-incomplete injuries), dynamometry testing can be an excellent means of objectively quantifying changes in isolated isometric and isokinetic muscular strength.

Upper extremity dynamometers may be more practical for people with SCI where spared upper extremity function is more likely and can be useful in providing objective measures of isolated upper extremity strength. Hand-held dynamometers, although limited in scope, may be useful in clinical settings where hand grip and pinch grip muscular strength can be assessed [69]. The more traditional and clinically accessible assessments of muscular strength and endurance consisting of 1RM and 10RM tests can be performed in different positions while testing muscles across single or multiple joints. Functional movements can also be performed and tested such as the modified push-up, modified pull-up, supine-to-sit, seated depression, and proneon-elbows press-up, to name a few. Pre- to post-comparisons can be made between the number of repetitions performed or amount of weight lifted before and after training. Trained therapists can also administer manually resisted muscular strength assessments in the form of a manual muscle test. The benefits of the manual muscle test include the ability to test single- and multi-joint strength, compare volitional strength between affected and non-affected limbs, and the ability of performing the testing in gravity minimized or eliminated positions. However, even among trained clinicians, manual muscle test scores are prone to subjectivity and results may be confounded by secondary factors such as spasticity, hypertonia, and structural limitations in joint range of motion. When selecting an appropriate muscular strength and endurance test, clinicians and exercise professionals should consider factors related to the level and severity of SCI, the muscle(s) being tested, and secondary factors that might bring into question the validity or reliability of the measures obtained.

\section{Muscle Strength and Endurance Training Strategies}

Once individualized baseline measures of muscular strength and endurance have been established, focused exercise training interventions can be employed. A variety of muscular strengthening strategies have been implemented in the SCI population. These include the use of free weights, elastic bands, plate-loaded machines, functional electrical stimulation, circuit training, body-weight resistance, and robotic technology. Many of these modalities have demonstrated some degree of positive effect on muscular strength and endurance; however, it is difficult to definitively interpret these findings given the heterogeneity in training mode, duration, intensity, and frequency used across studies. In two companion review articles examining the effects of exercise training on fitness parameters and the subsequent development of exercise guidelines based on these findings, the authors supported the benefits of participating in twice weekly resistance training ( 3 sets of $8-10$ repetitions) to improve muscular strength using free weights, elastic bands, plate-loaded machines, and functional electrical stimulation $[42,67 \cdot \bullet]$. This prescription is consistent with findings that a multi-day, multi-set resistance training program is effective for improving muscular strength and endurance of targeted muscles in persons with SCI [7, 39, 66, 70-76].

To the clinician and patient/consumer, improvement in muscular strength and endurance is only of significance if some meaningful benefit can be derived such that a reduction in secondary injury risk, enhanced functional capability, or improved health can be realized. One functional example would be improved walking ability, and with the development of various locomotor training devices, increased attention has been given to the combined use of resistance training and locomotor training to impact walking performance among persons with SCI. A few studies have investigated the effects of resistance training on gait speed and have demonstrated clinically meaningful changes; however, it is unclear, given the small sample size, whether these improvements were a result of improved isolated lower extremity strength or other neurophysiological or behavioral factors [77, 78]. The use of robotic-assisted locomotor training technology to improve functional muscular strength may have greater transference of effects than isolated resistance training programs alone. Research investigating the impact of robotic exoskeletons on muscular strength and other physical conditioning parameters are beginning to emerge, but additional research is needed to identify if any benefits might exist with this technology [79-84].

\section{Cardiorespiratory Fitness}

Relative to other health-related components of fitness, cardiorespiratory fitness has generated greater attention from both the clinical and research communities because poor cardiorespiratory fitness has been directly linked to the presence of secondary health conditions including cardiovascular disease and cardiometabolic syndrome in the SCI population $[40,85,86]$. Cardiorespiratory fitness, as a predictor of disease risk, has significant health implications for those with SCI and can be affected by a number of factors including level of injury, severity of injury, degree of physical deconditioning, and extent of ANS impairment. Various cardiorespiratory assessment methods 
and training strategies have been considered for persons with SCI.

\section{Cardiorespiratory Assessment Methods}

Cardiorespiratory fitness, as indicated by peak aerobic capacity or peak oxygen consumption $\left(\dot{V} \mathrm{O}_{2 \text { peak }}\right)$, can be determined using either direct or indirect methods. Although a number of predictive equations exist for determining $\dot{V} \mathrm{O}_{2 \text { peak }}$, metabolic testing using inspiratory and expiratory gas analysis is considered the gold standard and has been validated for use in healthy and clinical populations. Clinically meaningful cardiorespiratory biometrics can be obtained from direct measures of metabolic function including $\dot{V} \mathrm{O}_{\text {2peak }}$, heart rate (HR), respiratory exchange ratio (RER), minute ventilation $\left(\mathrm{V}_{\mathrm{E}}\right)$, and anaerobic threshold (AT). One must keep in mind that most individuals with SCI will not achieve a true maximum $\dot{V} \mathrm{O}_{2}$ $\left(\dot{V} \mathrm{O}_{2 \max }\right)$ as defined by the attainment of at least two out of three of the following criteria: [1] a plateau in $\mathrm{VO}_{2}$ with increase in external work load, [2] a maximal RER of $\geq 1.1$, and [3] a maximum heart rate within 10 beats/min of the age-predicted maximum [87]. $\dot{V} \mathrm{O}_{2 \max }$ is difficult to achieve for persons with SCI due to the reliance on a relatively smaller muscle mass to perform an exercise test (i.e., arm cycle ergometry), potential cardiovascular dysfunction caused by disruption of the ANS, impaired pulmonary function resulting in lower ventilator capacity [3032, 88, 89], or cardiovascular medications limiting HR.

Prior to performing any cardiorespiratory fitness assessment, it is necessary to obtain a pre-exercise health history and a basic functional assessment in order to screen for any existing relative or absolute contraindications to exercise testing, to establish which exercise test would be most appropriate (e.g., Bruce protocol), and to determine what types of assistive devices or modifications might be needed prior to testing $[32,90,91]$. Additional consideration should be given to factors such as level of injury (paraplegia vs. tetraplegia), degree of motor completeness, and neurological level of injury (ANS impairment). Once these considerations have been taken into account, clinicians and exercise professionals can appropriately choose from a number of cardiorespiratory testing modalities including wheelchair propulsion (treadmill or overground), volitional cycle ergometry (upper extremity, lower extremity or combined), electrically stimulated ergometry (upper extremity, lower extremity, or combined), overground ambulation, or treadmill ambulation. Clinicians and exercise professionals may choose to conduct either a submaximal or maximal exercise test. Typically, arm and leg cycle ergometry, wheelchair propulsion on a treadmill, and treadmill ambulation are performed using an incremental multistage test protocol where resistance is increased every $2-3$ min by $10-30$ watts per stage $[43,75$, $92,93]$. Guidelines for cardiorespiratory exercise test termination have been established by the American College of Sports Medicine (ACSM) and can be found in the Resource Manual for Guidelines for Exercise Testing and Prescription [43]. Results obtained from the exercise test can be used to determine relative risk for the development of secondary health conditions, to establish the efficacy of exercise interventions, and to assist in the development and implementation of the exercise prescription to optimize exercise training.

\section{Cardiorespiratory Intervention Strategies}

Numerous intervention strategies have been implemented by clinicians and researchers with the hope of positively affecting cardiorespiratory fitness among persons with SCI. These interventions include functional electrical stimulation (FES)-augmented training, volitional aerobic and circuit training, and robotic-assisted activity training. With each of these interventions, the goal is to place enough physical stress on the cardiovascular, pulmonary, and metabolic systems to induce sufficient overload and training effect. The ability to achieve these goals is highly dependent on the type of activity, the frequency, duration, and intensity of the activity, and the current degree of neurological and physiological impairment. It is promising to see that both clinicians and researchers are putting forth significant effort to better understand the complex interaction between these variables and are beginning to develop minimum physical activity requirements needed to achieve effective and meaningful change in cardiorespiratory fitness $[32,42,74,75,89,94 \bullet \cdot]$.

\section{FES-Augmented Training}

Exercise training using FES has received considerable attention in SCI because of its utility for those individuals with complete paralysis. This modality has been used in a variety of capacities including upper extremity FES-cycle ergometry, lower extremity FES-cycle ergometry, FESrowing, and combined upper and lower extremity FEShybrid exercise. Consistent conclusions are difficult given the heterogeneity of study protocols; however, current evidence suggests that changes in cardiorespiratory demand measured by $\dot{V} \mathrm{O}_{2}$ and other metabolic metrics can be achieved through the use of most FES modalities [9599]. In addition, there does appear to be additional metabolic benefit to FES-rowing and FES-hybrid exercise where higher $\dot{V} \mathrm{O}_{2 \text { peak }}$ values can be produced compared to upper extremity and lower extremity FES-cycle ergometry 
alone [95, 100-102]. Despite the higher $\dot{V} \mathrm{O}_{2 \text { peak }}$ observed in FES-rowing and FES-hybrid exercise, there is still insufficient or conflicting evidence whether the relative aerobic and metabolic demands of these activities are sufficient enough to bring about meaningful change in cardiorespiratory fitness. For example, the utility of FESaugmented exercise with respect to metabolic efficiency and demand has been questioned as the underlying mechanisms resulting in modest increases in $\dot{V} \mathrm{O}_{2 \text { peak }}$ may not sufficiently challenge the cardiorespiratory system to the extent needed for positive adaptation [103-105]. In the case of those with motor-complete SCI, this fact may be inconsequential as any opportunity to increase activity is likely better than none, and the added benefit of FESaugmented exercise as it relates to improved muscle structure and function can still be realized using this technology.

\section{Volitional Exercise}

Volitional exercise has been promoted extensively as a means of improving cardiorespiratory fitness and metabolic health in persons with SCI $[32,40,45,75,85,94,106]$ and have included arm cycle ergometry, upper extremity resistance training, wheelchair propulsion, swimming, and circuit resistance training, to name a few [45, 93, 102]. Exercise recommendations for cardiorespiratory training in SCI have been suggested by a number of organizations including the ACSM, the American Physical Therapy Association, and SCI Action Canada. Although some variation exists among the different recommendations in frequency, duration and intensity of aerobic exercise, the overall message of the various exercise prescriptions is consistent: [1] aerobic exercise is a necessary component needed to maintain or improve cardiorespiratory fitness, [2] aerobic exercise should be performed at a moderate to vigorous intensity corresponding with an increase in aerobic demand above the resting state, [3] continuous aerobic exercise should include a minimum of 20-30 min per session at least 2-3 times per week. Additional research must validate the effectiveness of these recommendations for SCI because compared to their able-bodied peers, they may require a greater volume of training to achieve meaningful improvements in cardiorespiratory fitness. Since individuals with higher level SCI will not necessarily have the means or functional capacity to participate in volitional exercise programs, separate recommendations may be needed.

\section{Robotic-Assisted Training}

Robotic exoskeleton devices are emerging technologies that have not been thoroughly investigated for their utility in improving physical conditioning and cardiorespiratory fitness following SCI. In 2013, Hoekstra et al. [107] investigated the effect of robotic-assisted gait training on cardiorespiratory fitness and compared the aerobic response to current recommended exercise guidelines for aerobic training. Only two out of ten subjects exercised at a capacity consistent with moderate intensity exercise $(>3$ METS) and no improvements in $\dot{V} \mathrm{O}_{2 \text { peak }}$ were observed after 24 training sessions. In two studies comparing relative cardiovascular response between robotic-assisted treadmill training and arm cycle exercise among persons with incomplete SCI, it was suggested that robotic-assisted treadmill training produced sufficient cardiorespiratory demand to potentially induce a positive training effect, but only when the individual actively engaged in the stepping motion during the session [108, 109]. In an exploratory study of overground ambulation using a robotic exoskeleton in a person with motor-complete SCI, only a modest increase in $\dot{V} \mathrm{O}_{2}$ relative to $\dot{V} \mathrm{O}_{2 \text { peak }}$ was observed which would not be sufficient to elicit a cardiorespiratory training effect [81]. Finally, a comparison of various training modalities including exoskeleton-, elliptical-, and therapist-assisted stepping revealed a lower oxygen consumption during exoskeleton-assisted stepping when compare to therapist-assisted stepping [110]. Despite the mixed cardiorespiratory responses observed in these studies, future technologies that combine FES and robotic exoskeletons may have a more significant impact on walking speeds, cardiorespiratory responses, and volitional movement during the activity. The allure of robotic-assisted exercise is also strong for those individuals with motor-complete tetraplegia where the long-term cardiorespiratory benefits from participation in FES-augmented and volitional exercise are still challenging.

\section{Conclusion}

Healthcare professionals are in the ideal position to positively affect health outcomes among persons with SCI by promoting exercise throughout the continuum of care from the hospital to the community to encourage lifelong healthy lifestyles. Fortunately, there is a growing interest and need from a public health perspective for clinicians, researchers, hospital administrators, and health insurance providers to develop and support strategies to minimize secondary health complications following debilitating injury. Current evidence indicates that both electrically stimulated and volitional exercise have the potential to improve components of fitness, reduce the risk of secondary health complications, and positively impact overall health for those with chronic physical disability. However, many 
challenges and questions remain regarding implementation and effectiveness of available fitness-promoting strategies.

Certainly some exercise is better than none, but given the complexity of SCI with respect to level and severity of injury, a clear consensus on the optimal frequency, duration, intensity, and mode of exercise needed to ensure longterm health benefits has yet to be determined. Additional consideration should be given to progression, overload, task specificity and activity-based activation of muscles below the injury level. Other considerations include injury risk reduction depending on exercise history, duration postinjury, and the presence of other factors such as fracture history, joint immobility, and ANS impairment. Furthermore, a number of unique physical, environmental, and psychosocial barriers to exercise participation exist and must be considered when developing exercise programs for persons with SCI. Thus, a much more individualized approach to exercise prescription may be warranted for people with SCI in order to realize meaningful fitness benefits and to justify the higher resource investment for access to specialized equipment, facilities, and staff. Finally, as is the case in non-injured populations, consideration should be given to both diet and exercise interventions, not simply exercise alone, when discussing optimal strategies for improving body composition and cardiovascular health for persons with SCI. Although research is emerging in this area, additional work must be done and collaboration with nutritionists/dieticians who specialize in SCI is needed in order to understand the unique relationship between these factors.

\section{Compliance with Ethics Guidelines}

Conflict of Interest SA Sisto and N Evans both declare no conflicts of interest.

Human and Animal Rights and Informed Consent This article does not contain any studies with human or animal subjects performed by any of the authors.

\section{References}

Papers of particular interest, published recently, have been highlighted as:

- Of importance,

•- Of major importance

1. National Spinal Cord Injury Statistical C. Spinal cord injury facts and figures at a glance. J Spinal Cord Med. 2014;37(2):243-4.

2. Gorgey A, Gater D. Prevalence of obesity after spinal cord injury. Topics Spinal Cord Injury Rehabil. 2007;12(4):1-7.

3. Bauman WA, Spungen AM. Metabolic changes in persons after spinal cord injury. Phys Med Rehabil Clin N Am. 2000;11(1):109-40.

4. Bauman WA, Spungen AM. Carbohydrate and lipid metabolism in chronic spinal cord injury. J Spinal Cord Med. 2001;24(4):266-77.
5. von Groote PM, Giustini A, Bickenbach JE. Analysis and implementation of a World Health Organization health report: methodological concepts and strategies. Am J Phys Med Rehabil Assoc Acad Phys. 2014;93(1 Suppl 1):S12-26.

6. Steiner W, Ryser L, Huber E, Uebelhart D, Aeschlimann A, Stucki G. Use of the ICF model as a clinical problem-solving tool in physical therapy and rehabilitation medicine. Phys Ther. 2002;82:1098-107.

7. Nash M, van de Ven L, Johnson B. Effects of resistance training on fitness attributes and upper-extremity pain in middle-aged men with paraplegia. Arch Phys Med Rehabil. 2007;88(1):70-5.

8. Liang H, Chen D, Wang Y, Rimmer JH, Braunschweig CL. Different risk factor patterns for metabolic syndrome in men with spinal cord injury compared with able-bodied men despite similar prevalence rates. Arch Phys Med Rehabil. 2007;88(9): 1198-204.

9. Gater DR, Jr. Obesity after spinal cord injury. Phys Med Rehabil Clin N Am. 2007;18(2):333-51, vii.

10. Laughton GE, Buchholz AC, Martin Ginis KA, Goy RE, Group SSR. Lowering body mass index cutoffs better identifies obese persons with spinal cord injury. Spinal Cord. 2009;47(10): 757-62.

11. Maki KC, Briones ER, Langbein WE, Inman-Felton A, Nemchausky B, Welch M, et al. Associations between serum lipids and indicators of adiposity in men with spinal cord injury. Paraplegia. 1995;33(2):102-9.

12. Garshick E, Kelley A, Cohen SA, Garrison A, Tun CG, Gagnon $\mathrm{D}$, et al. A prospective assessment of mortality in chronic spinal cord injury. Spinal Cord. 2005;43(7):408-16.

13. Cotton BA, Pryor JP, Chinwalla I, Wiebe DJ, Reilly PM, Schwab $\mathrm{CW}$. Respiratory complications and mortality risk associated with thoracic spine injury. J Trauma. 2005;59(6):1400-7; discussion 7-9.

14. Walter JS, Sacks J, Othman R, Rankin AZ, Nemchausky B, Chintam R, et al. A database of self-reported secondary medical problems among VA spinal cord injury patients: its role in clinical care and management. J Rehabil Res Dev. 2002;39(1):53-61.

15. Hart N, Laffont I, de la Sota AP, Lejaille M, Macadou G, Polkey MI, et al. Respiratory effects of combined truncal and abdominal support in patients with spinal cord injury. Arch Phys Med Rehabil. 2005;86(7):1447-51.

16. Brown R, DiMarco AF, Hoit JD, Garshick E. Respiratory dysfunction and management in spinal cord injury. Respir Care 2006;51(8):853-68; discussion 69-70.

17. Laffont I, Durand MC, Rech C, De La Sotta AP, Hart N, Dizien $\mathrm{O}$, et al. Breathlessness associated with abdominal spastic contraction in a patient with $\mathrm{C} 4$ tetraplegia: a case report. Arch Phys Med Rehabil. 2003;84(6):906-8.

18. Le Foll-de Moro D, Tordi N, Lonsdorfer E, Lonsdorfer J. Ventilation efficiency and pulmonary function after a wheelchair interval-training program in subjects with recent spinal cord injury. Arch Phys Med Rehabil. 2005;86(8):1582-6.

19. Hoffman MD. Cardiorespiratory fitness and training in quadriplegics and paraplegics. Sports Med. 1986;3(5):312-30.

20. Nash MS, Jacobs PL, Johnson BM, Field-Fote E. Metabolic and cardiac responses to robotic-assisted locomotion in motorcomplete tetraplegia: a case report. J Spinal Cord Med. 2004;27(1):78-82.

21. Dunlop D, Song J, Arnston E, Semanik P, Lee J, Chang R, et al. Sedentary time in U.S. older adults associated with disability in activities of daily living independent of physical activity. J Phys Act Health. 2014. doi:10.1123/jpah.2013-0311.

22. Kesaniemi Y, Danforth E Jr, Jensen M, Kopelman P, Lefebvre $\mathrm{P}$, Reeder B. Dose-response issues concerning physical activity and health: an evidence-based symposium. Med Sci Sports Exerc. 2001;33(6 Supplement):S351-8. 
23. Biering-Sorenson B, Brunn Kristensen I, Kjaer M, BieringSorenson F. Muscle after spinal cord injury. Muscle Nerve. 2009;40:499-519.

24. Gorgey A, Dudley G. Skeletal muscle atrophy and increased intramuscualr fat after incomplete spinal cord injury. Spinal Cord. 2007;45:304-9.

25. Burnham R, Martin T, Stein R, Bell G, MacLean I, Steadward R. Skeletal muscle fibre type transformation following spinal cord injury. Spinal Cord. 1997;35:86-91.

26. Lotta S, Scelsi R, Alfonsi E, Saitta A, Nicolotti D, Epifani P, et al. Morphometric and neurophysiological analysis of skeletal muscle in paraplegic patients with traumatic cord lesion. Paraplegia. 1991;29:247-52.

27. West C, Wong S, Krassioukov A. Autonomic carduiovascular control in Paralympic athletes with spinal cord injury. Med Sci Sports Exerc. 2014;46(1):60-8.

28. - West C, Bellantoni A, Krassioukov A. Cardiovascular function in individuals with incomplete spinal cord injury: a systematic review. Topics Spinal Cord Injury Rehabil. 2013;19(4):267-78. Cardiovascular dysfunction in individuals with incomplete spinal cord injury: a systematic review. Topics Spinal Cord Injury Rehabil. 2013;19(4):267-78. This review article describes the relationships between cardiovascular function and neurological and autonomic completeness of injury. This article is important in providing a comprehensive overview of the effect of SCI on cardiovascular biometrics including heart rate and blood pressure and draws comparisons between acute and chronic injury.

29. •• Waever L, Fleming J, Mathias C, Krassioukov A. Disordered cardiovascular control after spinal cord injury. Handbook of Clinical Neurology. 109 (3rd Series). Amsterdam: Elsevier; 2012. p. 213-33. This chapter provides a comprehensive overview of the effect of SCI on cardiovascular control. This chapter highlights the cardiovascular responses to exercise after SCI.

30. West C, Campbell I, Shave R, Romer L. Resting cardiopulmonary function in paralympic athletes with cervical spinal cord injury. Med Sci Sports Exerc. 2012;44(2):323-9.

31. Battikha M, Sa L, Porter A, Taylor J. Relationship between pulmonary function and exercise capacity in individuals with spinal cord injury. Am J Phys Med Rehabil. 2014;93(5):413-21.

32. Nash M. Exercise as a health-promoting activity following spinal cord injury. J Neurol Phys Therapy. 2005;29(2):87-106.

33. Rimmer J, Riley B, Wang E, Rauworth A, Jurkowski J. Physical activity participation among persons with disabilities: barriers and facilitators. Am J Prev Med. 2004;26(5):419-25.

34. Heath G, Fentem P. Physical activity participation among persons with disabilities: a public health perspective. Exerc Sport Sci Rev. 1997;25:195-234.

35. Stapleton JN, Martin Ginis KA. Sex differences in theory-based predictors of leisure time physical activity in a population-based sample of adults with spinal cord injury. Arch Phys Med Rehabil. 2014. doi:10.1016/j.apmr.2014.03.021.

36. Cowan R, Nash M, Anderson-Erisman K. Perceived exercise barriers and odds of exercise participation among persons with SCI living in high-income households. Topics Spinal Cord Injury Rehabil. 2012;18(2):126-7.

37. Scelza W, Klapkjian C, Zemper E, Tate D. Perceived barriers to exercise in people with spinal cord injury. Am J Phys Med Rehabil. 2005;84(8):576-83.

38. D'Oliveira G, Figueiredo F, Passos M, Chain A, Bezerra F, Koury J. Physical exercise is associated with better fat mass distribution and lower insulin resistance in spinal cord injured individuals. J Spinal Cord Med. 2014;37(1):79-84.

39. Pelletier C. Incorporating physical activity into the rehabilitation process after spinal cord injury. Appl Physiol Nutr Metab. 2014;39(4):513.
40. - Nash M, Cowan R, Kressler J. Evidence-based and heuristic approaches for customization of care in cardiometabolic syndrome after spinal cord injury. J Spinal Cord Med. 2012;35(5):278-92. This review article describes a systematic approach for individualized cardiometabolic syndrome risk assessment and countermeasures to disease after SCI. This article is important in highlighting the need for a combined approach incorporating exercise, diet, and pharmacological interventions.

41. Foulon B, Lemay V, Ainsworth V, Martin Ginis K. Enhancing physical activity guidelines: a needs survey of adults with spinal cord injury and health care professionals. Adapt Phys Activ Q. 2012;29:329-45.

42. Martin Ginis K, Hicks A, Latimer A, Warburton D, Bourne C, Ditor D, et al. The development of evidence-informed physical activity guidelines for adults with spinal cord injury. Spinal Cord. 2011;49:1088-96.

43. Swain D, editor. ACSM's resource manual for guidelines for exercise testing and prescription. 7th ed. Philadelphia: Wolters Kluwer/Lippincott Williams and Wilkins; 2013.

44. Casperson C, Powell K, Christenson G. Physical activity, exercise, and physical fitness: definitions and distinctions for health-related research. Public Health Rep. 1985;100(2):126-31.

45. Devillard X, Rimaud D, Roche F, Calmels P. Effects of training programs for spinal cord injury. Annales de Readaptation et de Medicine Physique. 2007;50:4908-80.

46. Monroe M, Tataranni P, Pratley R, Manore M, Skinner J, Ravussin E. Lower daily energy expenditure as measured by a respiratory chamber in subjects with spinal cord injury compared to control subjects. Am J Clin Nutr. 1998;68(6):1223-7.

47. Libin A, Tinsley E, Nash M, Mendez A, Burns P, Elrod M, et al. Cardiometabolic risk clustering in spinal cord injury: results of exploratory factor analysis. Topics Spinal Cord Injury Rehabil. 2013;19(3):183-94.

48. Gupta N, White K, Sandford P. Body mass index in spinal cord injury: a retrospective study. Spinal Cord. 2006;44:92-4.

49. Heyward V, Wagner D. Applied body composition assessment. Champaign, Illinois: Human Kinetics; 2004.

50. Terson de Paleville D, Lorenz D, McCulloch J, Aslan S, Kloby M, Love M, et al. Development of protocols to estimate maximal oxygen consumption and body composition in individuals with spinal cord injury. J Fed Am Soc Exp Biol. 2014;28(1 Suppl):884.17.

51. Yarar-Fisher C, Chen Y, Jackson A, Hunter G. Body mass index underestimates adiposity in women with spinal cord injury. Obesity. 2013;21(6):1223-5.

52. Beck LA, Lamb JL, Atkinson EJ, Wuermser LA, Amin S. Body composition of women and men with complete motor paraplegia. J Spinal Cord Med. 2013. doi:10.1179/2045772313Y. 0000000151.

53. Mojtahedi M, Valentine R, Evans E. Body composition assessment in athletes with spinal cord injury: comparison of field methods with dual-energy X-ray absorptiometry. Spinal Cord. 2009;47:698-704.

54. Maggioni M, Bertoli S, Margonato V, Merati G, Veicsteinas A, Testolin G. Body composition assessment in spinal cord injury subjects. Acta Diabetol. 2003;40:S183-6.

55. Singh R, Rohilla R, Saini G, Kaur K. Longitudinal study of body composition in spinal cord injury patients. Indian $\mathrm{J}$ Orthop. 2014;48(2):168-77.

56. Giangregorio L, McCartney N. Bone loss and muscle atrophy in spinal cord injury: epidemiology, fracture prediction, and rehabilitation strategies. J Spinal Cord Med. 2006;29(5):489-500.

57. Spungen A, Adkins R, Stewart C, Wang J, Pierson R, Waters R, et al. Factors influencing body composition in persons with spinal cord injury: a cross-sectional study. J Appl Physiol. 2003;95:2398-407. 
58. Kocina P. Body composition of spinal cord injured adults. Sports Med. 1997;23(1):48-60.

59. McQueen M. Exercise aspects of obesity treatment. Ochsner J. 2009;9:140-3.

60. Tanhoffer RA, Tanhoffer A IP, Raymond J, Hills AP, Davis GM. Exercise, energy expenditure and body composition in people with spinal cord injury. J Phys Act Health. 2013.

61. Gorgey A, Dolbow D, Cifu D, Gater D. Neuromuscular electrical stimulation attenuates thigh skeletal muscles atrophy but not trunk muscles after spinal cord injury. J Electromyogr Kinesiol. 2013;23(4):977-84.

62. Carty A, McCormack K, Coughlan G, Crowe L, Caulfield B. Alterations in body composition and spasticity following subtetanic neuromuscular electrical stimulation training in spinal cord injury. J Rehabil Res Dev. 2013;50(2):193-202.

63. Mahoney E, Bickel C, Elder C, Black C, Slade J, Apple D, et al. Changes in skeletal muscle size and glucose tolerance with electrically stimulated resistance training in subjects with chronic spinal cord injury. Arch Phys Med Rehabil. 2005;86(7):1502-4.

64. Ryan T, Brizendine J, Backus D, McCully K. Electrically induced resistance training in individuals with motor complete spinal cord injury. Arch Phys Med Rehabil. 2013;94(11):2166-73.

65. Giangregorio L, Craven C, Richards K, Kapadia N, Hitzig S, Masani K, et al. A randomized trial of electrical stimulation for walking in spinal cord injury: effects on body composition. J Spinal Cord Med. 2012;35(5):351-60.

66. Serra-Ano P, Pellicer-Chenoll M, Garcia-Masso X, Morales J, Giner-Pascual M, Gonzalez L-M. Effects of resistance training on strength, pain and shoulder functionality in paraplegics. Spinal Cord. 2012;50:827-31.

67. • Hicks A, Martin Ginis K, Pelletier C, Ditor D, Foulon B, Wolfe, DL. The effects of exercise training on physical capacity, strength, body composition and functional performance among adults with spinal cord injury: a systematic review. Spinal Cord. 2011;49:1103-27. This article provides a systematic review of evidence surrounding the effects of exercise on fitness in persons with SCI. This article is important in defining specific exercise recommendations for improved aerobic capacity and muscular strength based on results from current literature.

68. Khalil R, Gorgey A, Janisko M, Dolbow D, Moore J, Gater D. The role of nutrition in health status after spinal cord injury. Aging Dis. 2013;4(1):14-22.

69. Sisto SA, Dyson-Hudson T. Dynamometry testing in spinal cord injury. J Rehabil Res Dev. 2007;44(1):123-36.

70. Sasso E, Backus D. Home-based circuit resistance training to overcome barriers to exercise for people with spinal cord injury: a case study. J Neurol Phys Therapy. 2013;37(2):65-71.

71. Turbanski S, Schmidtbleicher D. Effects of heavy resistance training on strength and power in upper extremities in wheelchair athletes. J Strength Cond Res. 2010;24(1):8-16.

72. Harvey L, Fornusek C, Bowden J, Pontifex N, Glinsky J, Middleton $\mathrm{J}$, et al. Electrical stimulation plus progressive resistance training for leg strength in spinal cord injury: a randomized controlled trial. Spinal Cord. 2010;48:570-5.

73. Sabatier M, Stoner L, Mahoney E, Black C, Elder C, Dudley G, et al. Electrically stimulated resistance training in SCI individuals increases muscle fatigue resistance but not femoral artery size or blood flow. Spinal Cord. 2006;44:227-33.

74. Myslinski M. Evidence-based exercise prescription for individuals with spinal cord injury. J Neurol Phys Therapy. 2005;29(2): 104-6.

75. Jacobs P, Nash M. Exercise recommendations for people with spinal cord injury. Sports Med. 2004;34(11):727-51.

76. Jacobs P, Nash M, Rusinowksi J. Circuit training provides cardiorespiratory and strength benefits in persons with paraplegia. Med Sci Sports Exerc. 2001;33:711-7.
77. Gorgey A, Poarch H, Miller J, Castillo T, Gater D. Locomotor and resistance training restore walking in elderly person with a chronic incomplete spinal cord injury. Neuro Rehabilit. 2010;26(2):127-33.

78. Gregory C, Bowden M, Jayaraman A, Shah P, Behrman A, Kautz S, et al. Resistance training and locomotor recovery after spinal cord injury: a case series. Spinal Cord. 2007;45:522-30.

79. del-Ama A, Gil-Agudo A, Pons J, Moreno J. Hybrid gait training with an overground robot for people with incomplete spinal cord injury: a pilot study. Front Human Neurosci. 2014;8:Accessed Online.

80. Kozlowski A, Baeza Dager J, Firpi S, Tovar L, Voigt A, Dijkers $\mathrm{M}$, et al. Ekso-assisted walking for persons with spinal cord injury. Topics Spinal Cord Injury Rehabil. 2014;40(2 (Supplement 1)):S47.

81. Kressler J, Thomas CK, Field-Fote EC, Sanchez J, WiderströmNoga E, Cilien DC, et al. Understanding therapeutic benefits of overground bionic ambulation: exploratory case series in persons with chronic, complete spinal cord injury. Arch Phys Med Rehabil. 2014. doi:10.1016/j.apmr.2014.04.026.

82. Fleerkotte BM, Koopman B, Buurke JH, van Asseldonk EH, van der Kooij H, Rietman JS. The effect of impedance-controlled robotic gait training on walking ability and quality in individuals with chronic incomplete spinal cord injury: an explorative study. J Neuroeng Rehabil. 2014. doi:10.1186/1743-0003-11-26.

83. Jayaraman A, Thompson C, Rymer W, Hornby T. Short-term maximal-intensity resistance training increases volitional function and strength in chronic incomplete spinal cord injury: a pilot study. J Neurol Phys Therapy. 2013;37(3):112-7.

84. Wu M, Landry J, Schmit B, Hornby T, Yen S. Robotic resistance treadmill training improves locomotor function in human spinal cord injury: a pilot study. Arch Phys Med Rehabil. 2012;93(5):782-9.

85. Cowan R, Nash M. Cardiovascular disease, SCI and exercise: unique risks and focused countermeasures. Disabil Rehabil. 2010;32(26):2228-36.

86. Bauman W, Spungen A. Disorders of carbohydrate and lipid metabolism in veterans with paraplegia or quadriplegia: a model of premature aging. Metabolism. 1994;43(6):749-56.

87. Astorino T, Robergs R, Farzaneh G, Marks D, Burns S. Incidence of the oxygen plateau at VO2 max during exercise testing to volitional fatigue. J Exerc Physiol. 2000;3(4):1-12.

88. Ravensbergen H, de Groot S, Post M, Slootman H, van der Woude L, Claydon V. Cardiovascular function after spinal cord injury: prevalence and progression of dysfunction during inpatient rehabilitation and 5 years following discharge. Neurorehabilitation Neural Repair. 2014;28(3):219-29.

89. Figoni S. Perspectives on cardiovascular fitness and SCI. J Am Parapleg Soc. 1990;13(4):63-71.

90. Figoni S, Kirtali B, Sasaki R. Neuromuscular disorders: spinal cord dysfunction. In: Myers J, Nieman D, Frey G, editors. ACSM's resources for clinical exercise physiology: musculoskeletal, neuromuscular, neoplastic, immunologic, and hematologic conditions. Baltimore: Wolters Kluwer/Lippinott Williams and Wilkins; 2010. p. 58-78.

91. Zehr E. Evidence-based risk assessment and recommendations for physical activity clearance: stroke and spinal cord injury. Appl Physiol Nutr Metab. 2011;36(Supplement 1):S214-31.

92. El-Sayed M, Younesian A. Lipid profiles are influenced by arm cranking exercise and training in individuals with spinal cord injury. Spinal Cord. 2005;43:299-305.

93. Nash M, Jacobs P, Mendez A, Goldberg R. Circuit resistance training improves the atherogenic lipid profiles of persons with chronic paraplegia. J Spinal Cord Med. 2001;24(1):2-9.

94. • Galea M. Spinal cord injury and physical activity: preservation of the body. Spinal Cord. 2012;50:344-51. This review 
article examines the effects of physical rehabilitation on health, fitness, and target systems below the level of injury following SCI. This article is important in describing the effectiveness of exercise interventions on impacting fitness parameters, circulation, body composition, bone, and neural function and provides recommendations for future research in each of these areas.

95. Hettinga D, Andrews B. Oxygen consumption during functional electrical stimulation-assisted exercise in persons with spinal cord injury: implications for fitness and health. Sports Med. 2008;38(10):825-38.

96. Fornusek C, Davis G. Cardiovascular and metabolic responses during functional electrical stimulation cycling at different cadences. Arch Phys Med Rehabil. 2008;89:719-25.

97. Janssen T, Pringle D. Effects of modified electrical stimulationinduced leg cycle ergometer training for individuals with spinal cord injury. J Rehabil Res Dev. 2008;45(6):819-30.

98. Wheeler G, Andrews B, Lederer R, Davoodi R, Natho K, Weiss $\mathrm{C}$, et al. Functional electrical stimulation-assisted rowing: increasing cardiovascular fitness through functional electrical stimulation rowing training in persons with spinal cord injury. Arch Phys Med Rehabil. 2002;83:1093-9.

99. Phillips W, Kiratli B, Sarkarti M, Weraarchakul G, Myers J, Franklin B. Effect of spinal cord injury on heart and cardiovascular fitness. Curr Probl Cardiol. 1998;23(11):641-716.

100. Davis G, Hamzaid N, Fornusek C. Cardiorespiratory, metabolic, and biomechanical responses during functional electrical stimulation leg exercise: health and fitness benefits. Artif Organs. 2008;32(8):625-9.

101. Mutton D, Scremin E, Barstow T, Scott M, Kunkel C, Cagle T. Physiologic responses during functional electrical stimulation leg cycling and hybrid exercise in spinal cord injured subjects. Arch Phys Med Rehabil. 1997;78:712-8.

102. Verellen J, Vanlandewijck Y, Andrews B, Wheeler G. Cardiorespiratory responses during arm ergometry, functional electrical stimulation cycling, and two hybrid exericse conditions in spinal cord injured. Disabil Rehabil Assist Technol. 2007;2(2):127-32.

103. Hunt K, Hosmann D, Grob M, Saengsuwan J. Metabolic efficiency of volitional and electrically stimulated cycling in ablebodied subjects. Med Eng Phys. 2013;35:919-25.

104. Hunt K, Fang J, Saengsuwan J, Grob M, Laubacher M. On the efficiency of FES cycling: a framework and systematic review. Technol Health Care. 2012;20:395-422.

105. Barstow T, Scremin A, Mutton D, Kunkel C, Cagle T, Whipp BJ. Peak and kinetic cardiorespiratory responses during arm and leg exercise in patients with spinal cord injury. Spinal Cord. 2000;38:340-5.

106. Fernhall B, Heffernan K, Young Jae S, Hedrick B. Health implications of physical activity in individuals with spinal cord injury: a literature review. J Health Human Services Adm. 2008;30(4):468-502.

107. Hoekstra F, van Nunen M, Gerrits K, Stolwijk-Swuste J, Crins $\mathrm{M}$, Janssen T. Effect of robotic gait training on cardiorespiratory system in incomplete spinal cord injury. J Rehabil Res Dev. 2013;50(10):1411-22.

108. Jack LP, Purcell M, Allan DB, Hunt KJ. The metabolic cost of passive walking during robotics-assisted treadmill exercise. Technol Health Care Off J Eur Soc Eng Med. 2011;19(1): 21-7.

109. Jack LP, Purcell M, Allan DB, Hunt KJ. Comparison of peak cardiopulmonary performance parameters during roboticsassisted treadmill exercise and arm crank ergometry in incomplete spinal cord injury. Technol Health Care Off J Eur Soc Eng Med. 2010;18(4-5):285-96.

110. Hornby T, Kinnaird C, Holleran C, Rafferty M, Rodriguez K, Cain J. Kinematic, muscular, and metabolic responses during exoskeletal-, elliptical-, or therapist-assisted stepping in people with incomplete spinal cord injury. Phys Ther. 2012;92(10):1278-91. 